\title{
Regeneration, Stem Cells, and the Evolution of Tumor Suppression
}

\author{
B.J. Pearson AND A. SÁnChez Alvarado \\ Howard Hughes Medical Institute, Department of Neurobiology and Anatomy, \\ University of Utah School of Medicine, Salt Lake City, Utah 84132
}

\begin{abstract}
All multicellular organisms have requirements for tumor suppression to regulate cellular proliferation during either embryonic development or adult life. However, different organisms have vastly different requirements. Adult tumor suppression is probably not crucial to organisms possessing both short life spans and largely postmitotic soma. In contrast, animals with lifelong tissue turnover or those capable of regenerating body parts lost to injury must possess evolutionarily selected mechanisms to control rates of cell proliferation such that tissue homeostasis can be maintained or restored after injury. We hypothesize that these biological differences may help to explain why the lists of tumor suppressor genes in humans and Drosophila are largely nonoverlapping. Here, we address this disparity by examining the tumor suppressor gene content of two outgroups to the vertebrates and flies/nematodes: the freshwater planarian and the single-celled choanoflagellate. Both of these organisms have recently had their genomes sequenced, giving us a first glimpse of which known tumor suppressor genes have been maintained during evolution. In addition, we attempt to resolve which genes may have had ancestral tumor suppressor function and which may have acquired this function de novo.
\end{abstract}

Of all the genes that can contribute to the exceedingly complex disease known as "cancer," perhaps the most amenable to functional dissection are the tumor suppressor genes (TSGs). Simply defined, a gene is a tumor suppressor if it can meet several criteria: (1) When it is removed from an organism, it results in a tumor or hyperproliferation phenotype, (2) mutations in these genes are mostly recessive and function cell-autonomously, and (3) the given gene must also be mutated or deleted in human cancers. A search of PubMed using the quoted phrase "tumor suppressor" retrieved 59,612 entries (as of October 7, 2008). Such a vast field of ongoing research into the functions of TSGs is driven by a desire to understand the ontogeny of human cancers and find new genes to target for therapy. However, it is important to remember that during evolution, TSGs were not selected because of their role in the disease of cancer. Instead, these genes likely evolved to function in indispensable cellular processes such as control of cell division and DNA-damage repair. Thus, the reason that these genes exist seems to be straightforward, but it does not explain the paradox that the major TSGs in human cancers are not TSGs in model laboratory invertebrates. Our collective lack of understanding of the evolution of TSGs may explain why a search of PubMed using the quoted phrases "tumor suppressor evolution," "evolution of tumor suppressors," or "evolution of tumor suppression" retrieved a total of zero entries. Thus, studies attempting to understand tumor suppression through the lens of evolutionary biology are uncommon.

There are approximately 50 million species of animals on our planet (Brusca and Brusca 2003). Of these, less than 10 have been used in the laboratory to understand the mechanisms of tumor suppression. Of these 10 , only 2 species have been used outside of the vertebrates and both are from the super-phylum Ecdysozoa, i.e., Drosophila melanogaster and Caenorhabditis elegans. Remarkably, our understanding of tumor development and the disparities that are known to exist among species comes from this small, not statistically significant sample size. This notable shortcoming in our knowledge imposes serious limitations in our interrogation of tumor suppression mechanisms. First, the general absence of molecular studies in other phyla leaves unanswered the question of how tumor suppression evolved. Second, when these facts are taken into consideration, the difficulties of postulating bona fide conclusions about how tumor suppression works become readily apparent. Therefore, mechanistic studies of tumor suppression in underrepresented, but evolutionarily important organisms are not only warranted, but also essential to fill this lacunae of knowledge.

Because regulation of cellular proliferation is indispensable to the survival of multicellular organisms, it is likely that mechanisms for tumor suppression have ancestral evolutionary origins. Equally important, physiological and environmental changes often require adult organisms to modulate their rates of cell proliferation (e.g., regeneration of body parts lost to injury), and therefore mechanisms to relax tumor suppression in order to allow temporary hyperproliferation must have also evolved. To investigate these hypotheses, at least two questions need to be answered: Did the unicellular ancestor to animals already have tumor suppressor genes that were then recruited into tumor suppression? Or, did animals evolve tumor suppressor genes de novo to contend with survival and perpetuation issues raised by the emergence of multicellularity? 
In this chapter, we investigate the evolutionary implications of tumor suppression. First, we explore the different types of tumor suppression requirements in various organisms with the objective of determining whether universal tumor suppressors exist in the Metazoa. Second, we examine the complement of known, well-studied tumor suppressor gene homologs across animals, including the newly sequenced genomes of the freshwater planarian Schmidtea mediterranea and the unicellular choanoflagellate Monosiga brevicollis (King et al. 2008). We then compare and contrast the functions of common TSGs in flies, C. elegans, and vertebrates and attempt to answer why differences exist while tracking these genes throughout the evolution of animals. Finally, we argue that the freshwater flatworm (planaria) can be used not only to reconcile evolutionary hypotheses, but also to offer a fresh view on tumor suppression mechanisms.

\section{TYPES OF TUMOR SUPPRESSION VERSUS LIFE SPAN AND ADULT CELL PROLIFERATION}

All animals have requirements for tumor suppression, but animal tumors can be generally cataloged as either embryonic/larval (arising during development) or late-onset (arising in adults). The type most prevalent in different organisms sharply correlates with life span and somatic cell proliferation during adulthood. For example, in animals possessing few adult somatic cell divisions and a short life span, adult tumor suppression is not nearly as important as tumor suppression during embryonic/larval development, where extensive cell proliferation is the norm.

In Drosophila and C. elegans, it is clear that their susceptibility to tumors is high during embryonic/larval development (e.g., scribbled [scrib], lethal giant larvae [lgl], lethal giant discs [lgd], discs large [dlg], malignant brain tumor [mbt], brain tumor [brat]) (Gateff and Schneiderman 1974; Xu et al. 1995; Woodhouse et al. 1998; Humbert et al. 2003; Klezovitch et al. 2004; Menut et al. 2007). This hypothesis is supported by the fact that no tumor suppressor mutation has yet been found in Drosophila or C. elegans to specifically affect adults (i.e., not arise during development). Even though no adult-onset tumors have been identified in these organisms, the TSGs that function during Drosophila development, for example, have extensively informed cell biological processes involved in vertebrate tumorigenesis and metastasis.

In principle, vertebrates have developmental tumor suppression requirements similar to those of flies and C. elegans. However, we hypothesize that vertebrates have fundamentally different requirements for adult tumor suppression due to notable differences in life span and the numbers of adult somatic cell divisions (Fig. 1A). For instance, flies and $C$. elegans have very short adult life spans (60 and 14 days, respectively) and therefore might not require robust adult tumor suppression. In contrast to these two invertebrates, the shortest captive life span documented for a vertebrate is 90 days (Terzibasi et al. 2007), but most vertebrates live 10-100 times longer than Drosophila. Furthermore, flies and C. elegans have relatively few adult somatic cell divisions (homeostasis) (0 and likely $\sim 10^{5}$, respectively), which may not be enough cell turnover to select for the maintenance of tumor suppressor function, especially when coupled to their short life spans. Humans, on the other hand, are predicted to turn over many of the approximately $10^{14}$ cells in our bodies throughout our lives, leading to homeostasis of about $10^{10}$ cells per day (Heemels 2000). That is $10^{7}$ times higher than the number of cells present in an entire adult $C$. elegans and $10^{5}$ more cells than a fly turns over in a lifetime. With these staggering differences in cell turnover, it is easy to hypothesize that natural selection may have selected for completely different tumor suppression mechanisms in vertebrates. The sheer number of constant cell turnover in humans, for example, must be under tight genetic control and simultaneously must require constant tumor suppression activity. Consistent with this observation is the fact that many adult TSGs in vertebrates do not appear to function as adult tumor suppressors in Drosophila or C. elegans (phosphatase and tensin homolog deleted on chromosome 10 [PTEN], p53, retinoblastoma [Rb], neurofibromatosis 1 [NF1], p16 ${ }^{\text {ink } 4 a}, p 19^{A R F}, p 21^{\text {cipl }}, p 27^{\text {Kipl }}$, breast cancer susceptibility gene 1 [Brcal], breast cancer susceptibility gene 2 [Brca2], deleted in lung cancer 1 [DLC1]) (de Nooij et al. 1996; The et al. 1997; Huang et al. 1999; Sherr and McCormick 2002; Stevaux et al. 2002; Coqueret 2003; Lowe and Sherr 2003; Carroll and Stone-
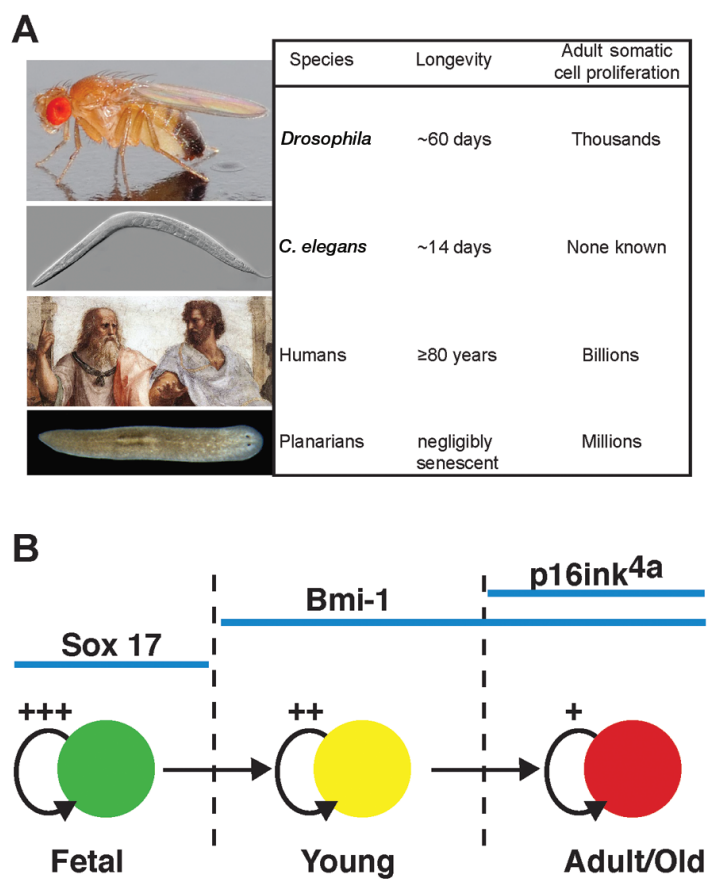

Figure 1. Cell proliferation and animal longevity. $(A)$ Comparison of longevity and cell proliferative activities between humans and established (C. elegans, Drosophila) and emerging organisms $(S$. mediterranea). ( $B$ ) Expression of known activators and suppressors of neuronal stem cells throughout mammalian life. Expression of the Polycomb group gene Bmi-1 is continuously maintained after birth. As animals age, however, expression of the tumor suppressor gene $p 16^{\text {inkta }}$ is observed, resulting in an inhibition of stem cell self-renewal (Molofsky et al. 2006). (Drosophila, nematode, and human images courtesy of http://commons.wikimedia.org.) 
cypher 2005; Evers and Jonkers 2006; Lu and Abrams 2006; Rossi and Weissman 2006; Aoki and Taketo 2007; Moon et al. 2008; Xue et al. 2008).

Many of the old cells replaced during homeostasis in mammals are produced by tissue-specific adult stem cells. Remarkably, during all of these divisions, the adult stem cells strictly maintain their numbers. Implied in this is that there are genetic mechanisms to constantly prevent hypoproliferation (organismal senescence) or hyperproliferation (tumorigenesis) that exist specifically in adult stem cells themselves. Evidence for the existence of such mechanisms is provided by the age-dependent activation of TSGs as neuronal stem cells age in mammals (Fig. 1B). Young neuronal stem cells have been shown to depend on the function of the Polycomb group gene Bmi-1 for their self-renewal. When this gene is eliminated, cell proliferation decreases and a marked increase in the expression of the tumor suppressor $p 16^{\text {Ink4a }}$ is observed. This observation suggests that Bmi-1 may actually repress tumor suppression in order to promote stem cell proliferation in young individuals (Molofsky et al. 2003). Interestingly, as animals age and the proliferative capacities of neural stem cells in the subventricular zone and olfactory bulb all decline, a concomitant increase in the expression of $p 16^{\text {Ink4a }}$ is observed (Molofsky et al. 2006). These findings suggest that regulation of proliferation of somatic stem cells may have been selected through evolution to balance the longevity assured by stem-cell-dependent tissue renewal against the risk of developing hyperproliferative disorders such as cancer (Gatza et al. 2007).

\section{PLANARIANS AND CHOANOFLAGELLATES AS NEW ORGANISMS TO UNDERSTAND TUMOR SUPPRESSION}

In contrast to flies and nematodes, the adult freshwater planarian $S$. mediterranea possesses an abundant and experimentally accessible population of adult somatic stem cells known as neoblasts (Newmark and Sánchez Alvarado 2002). Many of these cells are continuously undergoing cell division (Newmark and Sánchez Alvarado 2000), and their progeny become determined and ultimately differentiate into all of the known cell types in planarians (Newmark and Sánchez Alvarado 2000; Eisenhoffer et al. 2008). In fact, planarians derive their well-known tissue homeostasis and regenerative properties from regulating the proliferation and functions of neoblasts and their division progeny (Reddien and Sánchez Alvarado 2004; Sánchez Alvarado and Tsonis 2006). After amputation, for instance, planarians develop at the cut surface a specialized structure known as a regeneration blastema, which is the result of extensive proliferation by neoblasts, and which will ultimately differentiate to replace the missing body parts lost to injury. The fact that planarians can temporarily allow their stem cells to hyperproliferate after injury, only to reign in this activity to normal levels, indicates the existence of molecular mechanisms that are constantly surveying changes in the environment and orchestrating the appropriate changes in proliferation required by such changes. Therefore, an investigation of tumor suppression mechanisms in this invertebrate should help inform to what extent the molecular basis of tumor suppression may be evolutionarily conserved among multicellular organisms.

By the same token, the genetic composition of the closest unicellular relative of the multicellular animals, the choanoflagellate $M$. brevicollis, should provide us with a glimpse of the molecular tool kit available before the emergence of multicellularity. Analyses of the Monosiga genome indicate that a large number of transcription factor families previously thought to be restricted to metazoa are found in this organism, as well as proteins involved in multicellularity, such as cadherins, transmembrane C-type lectins, and other molecules in cell adhesion (King et al. 2008). Therefore, determining the presence or absence of known tumor suppressors in the genome of this organism should provide evidence for either the ancestry or novelty of known tumor suppressor genes.

\section{EVOLUTIONARY SCENARIOS OF TUMOR SUPPRESSION}

On the basis of the above information, we pose three different hypotheses that can explain the disparity in tumor development known to exist between vertebrates and flies/C. elegans. First, the genome of the ancestor of flies, C. elegans, and vertebrates contained all of the homologs of tumor suppressor genes, but only embryonic/larval tumor suppression function was maintained in flies and nematodes (or lost in vertebrates). Second, the genome of the ancestor of flies, C. elegans, and vertebrates contained all of the gene homologs for tumor suppression, but only in the vertebrate lineage did they acquire adult tumor suppressor function (or lost in flies/C. elegans). Third, the genome of the ancestor of flies and vertebrates did not have all of the homologs of TSGs, and these emerged de novo and independently in both clades of animals, giving us the disparate list of genes discussed above. These various scenarios may be resolved by examining the first three TSGs identified in flies, and the top three, most frequently mutated TSGs in human cancers. We can then search the genomes of outgroup organisms (planarians and Monosiga) to determine whether they contain these genes and examine their functions if they are known.

\section{EVOLUTION OF TSGS IN DROSOPHILA: $S C R I B, L G L, A N D D L G$}

The embryonic/larval tumor suppressors in flies are referred to as neoplastic TSGs (nTSGs), and they were found in genetic screens in which larvae could not pupate or displayed imaginal disc overgrowth in conditional mutant screens (Gateff and Schneiderman 1974; Gateff and Mechler 1989; Gateff 1994; Xu et al. 1995; Pagliarini and Xu 2003; Menut et al. 2007). Analyses of these genes have shown that they function mainly in the establishment and maintenance of correct apical-basal cell polarity in proliferating epithelia and neuroblasts during fly development (Humbert et al. 2003; Vasioukhin 2006). When mutated, cells in developing epithelial structures hyperproliferate and can even metastasize when dissected and transplanted into the abdomen of another adult fly. Interestingly, if a 
developing tissue is only partially mutant for these nTSGs, the cells are often at a growth disadvantage and do not produce tumors nor metastasize. This observation is contrary to canonical mammalian tumorigenesis, in which the vast majority of human tumors are monoclonal, and the rare mutant cells for a TSG occur in an otherwise wild-type tissue (Hanahan and Weinberg 2000; Wang et al. 2007). Finally, it does not appear that vertebrate homologs of fly nTSGs function as tumor suppressors during development or in adults, although only several studies have been performed (see discussion). Because most human cancers also arise from epithelia and because human epithelia are also polarized, it seems reasonable to hypothesize that homologs of the fly nTSGs function in the later stages of cancer, when cellular polarity is commonly lost (Wodarz and Nathke 2007).

We traced the evolution of these nTSGs across the animal kingdom and our analyses led us to a few interesting conclusions (Fig. 2). First, there is similar gene redundancy in vertebrates, planaria, and Monosiga but, generally, single gene orthologs in flies and C. elegans. For instance, although flies have a (likely) single $d l g$, vertebrates, planaria, and Monosiga have at least four. This could explain the discrepancy of why individual vertebrate $d l g$ homologs do not appear to have tumor suppressor function in vertebrates. Another interesting conclusion is that the genome of unicellular choanoflagellates already have clear homologs to scrib and $d l g$, but not lgl. Because $s c r i b, l g l$, and $d l g$ are involved in the same structure of adherens and tight junctions in both invertebrates and vertebrates, it will be interesting to determine what their functions may be in choanoflagellates and what kind of complex Monosiga makes without the presence of $l g l$. The possibility exists that choanoflagellates use these genes in proliferation control in a manner similar to that of Drosophila. If shown to be true, this would mean that the tumor suppressor activity of the nTSGs was either lost in the vertebrates or not sufficiently and rigorously tested. Alternatively, a similar conclusion suggesting that the nTSGs had ancestral tumor suppressor activity would also be reached if these molecules are found to also be tumor suppressors in planarians.

\section{EVOLUTION OF TSGs IN VERTEBRATES: $P 53$, PTEN, AND RB}

Although there are many TSGs in vertebrates, virtually every cancer has a mutation in at least one of three genes: p53, PTEN, and Rb (Fig. 3). Of these three, none more perfectly illustrate the differences between invertebrates and vertebrates to develop tumors than p53. p53 is mutated in more than $50 \%$ of all human cancers (Harris 1996), yet p53 mutant Drosophila or C. elegans develop normally and are tumor free (Brodsky et al. 2000; Ollmann et al. 2000; Derry et al. 2001; Rong et al. 2002; Wells et al. 2006).

On the basis of gene number and protein function, p53 possesses a complex, if not tortuous, evolutionary history. Although Drosophila and C. elegans only have a single p53 family member, there has been an expansion of the family in many other organisms (Nedelcu and Tan 2007). Vertebrates have three family members: p53, p63, and p73, of which the latter two are not robust tumor suppressors and have an additional carboxy-terminal sterile $\alpha$ motif (SAM) (Lu and Abrams 2006). Furthermore, p63 and p73 have many spliceoforms that can act as dominant negatives to p53 (often making them oncogenes) ( $\mathrm{Li}$ and Prives 2007). The cross-regulation and redundant function of vertebrate p53 family members is exceedingly complex and can vary from tissue to tissue (Fei et al. 2002). Despite some differences in domain structure, the p53 family appears to have retained specific ancestral functions. For

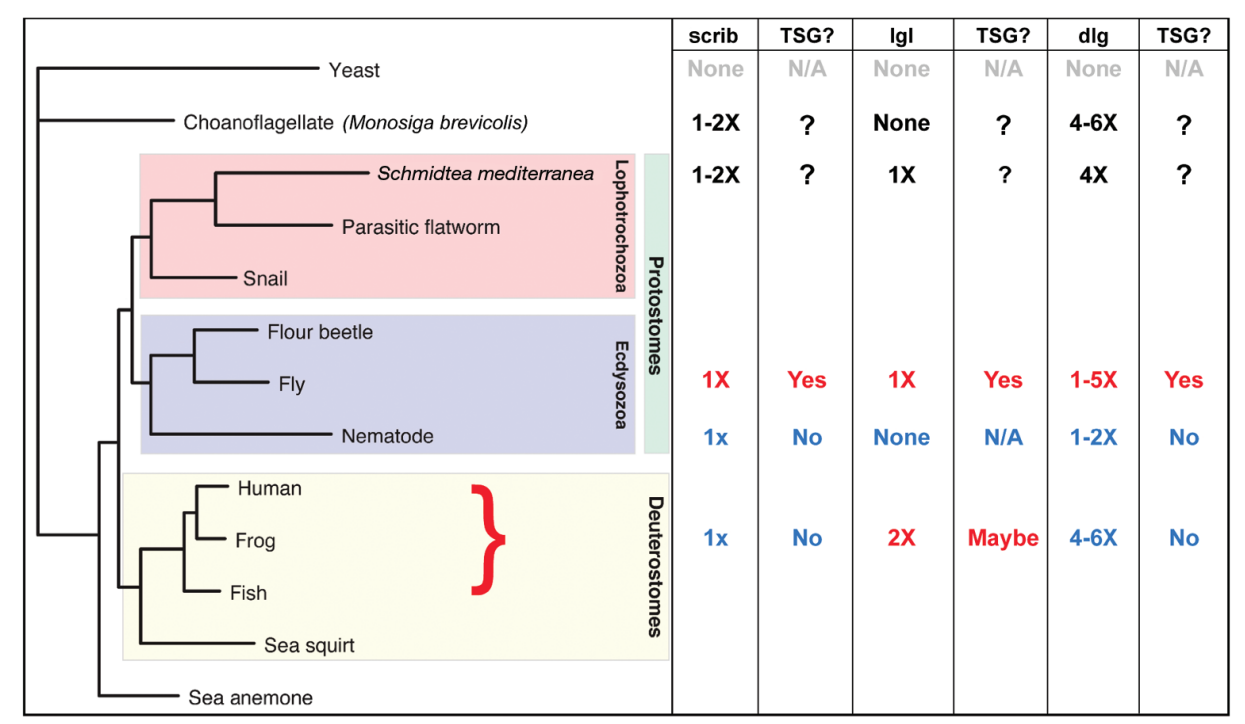

Figure 2. Evolution of the fly nTSGs scrib, lgl, and dlg. Reciprocal BLAST searches of the Drosophila neoplastic tumor suppressor genes scrib, $l g l$, and $d l g$ were performed against the genomes of choanoflagellates ( $M$. brevicollis), planaria ( $S$. mediterranea), nematodes (C. elegans), and vertebrates (fish, frog, and human). Phylogenetic relationships are illustrated on the left and the number of homologs found for each of the genes is listed. (Red text) Evidence for TSG function; (blue text) evidence for no TSG function; (black text) function unknown. 


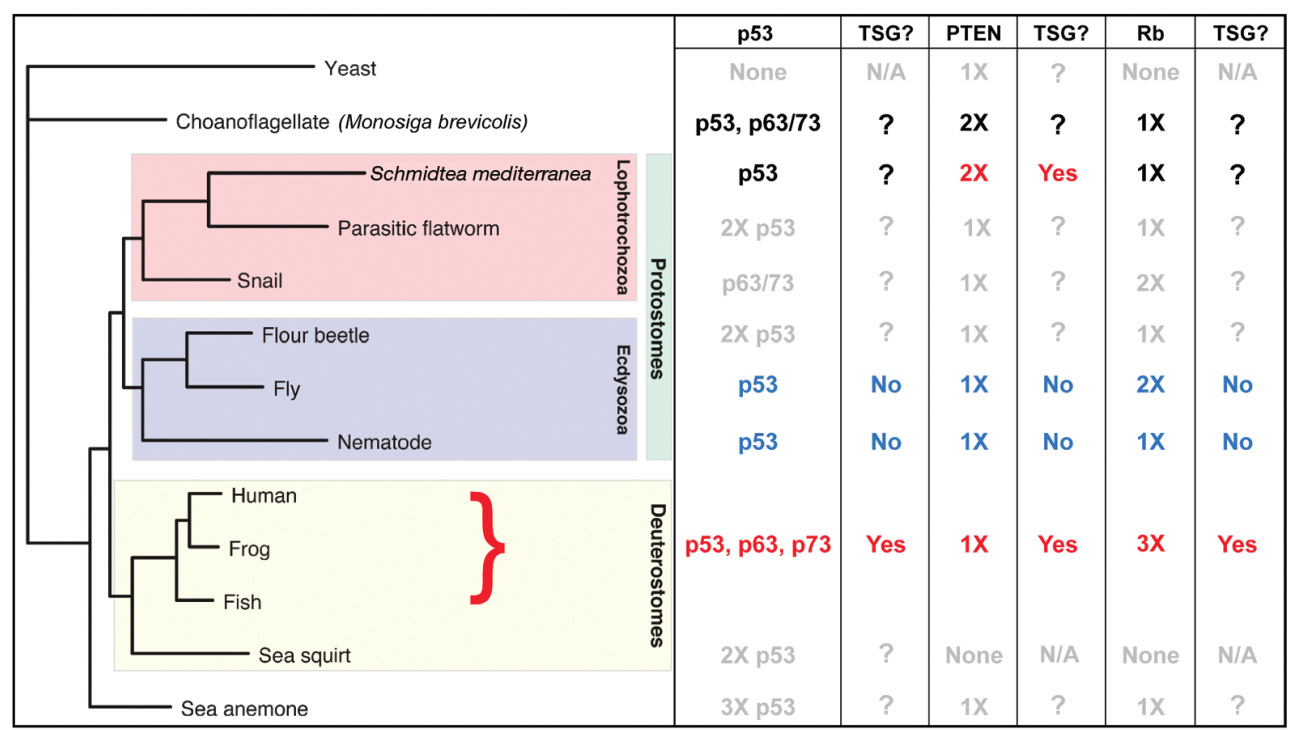

Figure 3. Evolution of the vertebrate TSGs p53, PTEN, and Rb. Reciprocal BLAST searches of the vertebrate tumor suppressor genes $p 53, P T E N$, and $R b$ were performed against the genomes of yeast (Saccharomyces cerevisiae, Schizoaccharomyces pombe), choanoflagellates (M. brevicollis), sea anemone (Nematostella vectensis), planaria (S. mediterranea), parasitic flatworms (Schistosoma mansoni), snails (Lottia gigantea), flour beetle (Tribollium castaneum), nematodes (C. elegans), urochordates (Ciona intestinalis), and vertebrates (fish, frog, and human). Phylogenetic relationships are illustrated on the left and the number of homologs found for each of the genes is listed in the table. Question marks indicate instances in which orthology could not be conclusively established. Text color coding is the same as that in Figure 2.

example, all three p53 family members in vertebrates respond to genotoxic stresses and can induce apoptosis (Cuddihy and Bristow 2004; Irwin 2004; MurrayZmijewski et al. 2006). Fly and C. elegans p53 also have this function and as such can be assigned as ancestral ( $\mathrm{Lu}$ and Abrams 2006). Apparent new functions for the p53 family in vertebrates are tumor suppression/cell cycle regulation ( $p 53)$, epithelial stem cell self-renewal ( $p 63)$ (Senoo et al. 2007), and neural differentiation and survival (p73) (Moll and Slade 2004; Rocco et al. 2006).

It is widely accepted that p53 has acquired tumor suppressor function in vertebrates from an ancestral role of DNA-damage sensing/repair. However, there has never been a functional study of a p53 family member in an invertebrate that has high requirements for adult tumor suppression, nor in an animal from the Lophotrochozoan superphylum. Planaria have a single homolog of p53, and once again, these organisms are in an advantageous position to resolve whether the "new" functions of the p53 family in vertebrates are actually new or ancestral (but lost in flies/C. elegans). Surprisingly, Monosiga has a homolog to both p53 and p63 (Nedelcu and Tan 2007). Determining how these genes function in a single-celled animal will almost certainly yield insights into the evolution of tumor suppressor activity of p 53 family members.

Unlike $\mathrm{p} 53$, the $\mathrm{Rb}$ transcription factor has a clear evolutionary history and an ancestral function in cell cycle regulation and was the first tumor suppressor to be identified (van den Heuvel and Dyson 2008). One of the three $R b$ genes in humans is thought to be mutated in virtually all cancers (Weinberg 1995; Ajioka et al. 2007). In vertebrates, $\mathrm{Rb}$ functions by repressing the expression and activation of the E2F family of proteins, which are needed for multiple processes during the cell cycle (van den Heuvel and Dyson 2008). Although no obvious homolog to $R b$ can be found in fungi, homologs of this gene can be found in plants and in unicellular (Monosiga) and multicellular animals. Flies have two Rb family members; however, one mutant shows only mild overgrowth defects (Du et al. 1996; Stevaux et al. 2002). Judging from the fact that Monosiga, C. elegans, planarians, and sea anemone (Nematostella vectensis) have a single $\mathrm{Rb}$ family member, this suggests that the ancestral condition in animals was a single gene. Interestingly, vertebrates, flies, and snails (Lottia gigantea) have expanded the family, and the urochordate Ciona intestinalis (sea squirt) has somehow lost this gene. Even though $\mathrm{Rb}$ is the closest example of a universal TSG that we cover in this chapter, its function has not been examined in planaria or Monosiga, so once again the sample size for making bona fide conclusions is small. We predict that $\mathrm{Rb}$ will function as a negative cell cycle regulator in planarians, Monosiga, and other metazoans.

The third most mutated gene in human cancers is PTEN. PTEN functions to balance levels of the intracellular signaling molecule phosphatidylinositol-3-phosphate ( $\mathrm{PIP}_{3}$ ) with the gene phosphoinositol-3 kinase (PI3K), and has crucial roles in cellular proliferation, differentiation, and migration in both vertebrates and invertebrates (Sulis and Parsons 2003). Mutations of this gene are found in a wide variety of cancers, including non-Hodgkin's lymphoma (Nakahara et al. 1998), breast (Kirkegaard et al. 2005), prostate ( $\mathrm{Li}$ et al. 1997), and some types of pancreatic cancers (Asano et al. 2004). Altogether, this body of evidence suggests that PTEN may have a key role in regulating and misregulating tissue homeostasis in mammals (Marx 2007). Although the PTEN signaling pathway has many loops, intersections, and forks (Sulis and Parsons 2003), it is generally thought that in PTEN mutants, the canonical 
PI3K-Akt-mTOR (mammalian target of rapamycin) pathway is the dominant route. This is supported by suppression of PTEN-mediated tumorigenesis by the small molecule rapamycin, which is thought to inhibit mTOR. In mice, for instance, rapamycin treatment compensates for PTEN loss and is effective in preventing leukemia-initiating cells, while keeping normal hematopoietic function in mice with a PTEN conditional deletion (Yilmaz et al. 2006).

Similar to p53, elimination of PTEN in Drosophila does not result in defects associated with tumor suppression (Huang et al. 1999). Instead, the PTEN mutant phenotype results in defects associated with the scale and growth of cells rather than with cellular proliferation. For example, a PTEN mutant eye imaginal disc in Drosophila does not show hyperproliferation, even though cells in the disc are enlarged and/or misshapen (Huang et al. 1999). On the basis of gene complement, it is likely that the ancestral condition was a single PTEN gene which was expanded to two genes in Monosiga and planarians and, again, surprisingly lost in the sea squirt (C. intestinalis). Unlike p53 and $\mathrm{Rb}$, a PTEN-like gene does exist in the fungi. Vertebrates also have two closely related genes - TPIP and TPTEthat have no fly homologs. Interestingly, planaria have a single homolog to TPIP/TPTE that appears to be the ancestral condition. Therefore, the gene complement for the PTEN homologs in planaria appears to be much more similar to vertebrates.

Recently, we analyzed the function of two planarian PTEN orthologs (Oviedo et al. 2008). When both SmedPTEN1 and Smed-PTEN2 are inhibited by RNA interference (RNAi), adult planaria show ectopic growths, hyperproliferation of stem cells, and breakdown of the subepithelial basement membrane that surrounds the worm (Fig. 4). Interestingly, rapamycin can suppress all three of these phenotypic outcomes (Oviedo et al. 2008). These results are the first to show that PTEN can function as a tumor suppressor in invertebrates. In addition, this discovery demonstrates that planaria can be used as a model to understand the early stages of tumorigenesis as well as to interrogate the function of tumor suppressor genes in adult animals. In the context of this review, these data suggest that the ancestral function of PTEN in multicellular animals was also in the tumor suppression that flies and C. elegans have lost.

\section{DISCUSSION}

Despite differences in tumor suppression between flies/C. elegans and vertebrates/planaria, it is clear that more experiments testing the ideas presented in this chapter are needed. First, it has recently been shown that Drosophila does indeed possess adult stem cells (Micchelli and Perrimon 2006). In addition, now that a cellular lineage has been at least partially elucidated for fly intestinal antibody-secreting cells (ASCs), it is possible to test what happens to these cells when $p 53, P T E N$, or $R b$ are conditionally deleted in adults. It is formally possible that deletion of these TSGs will give rise to hyperproliferation and expansion of the ASC population, further cementing the ancestral role of these genes in tumor suppression. Because planarians have already been shown to be amenable to the

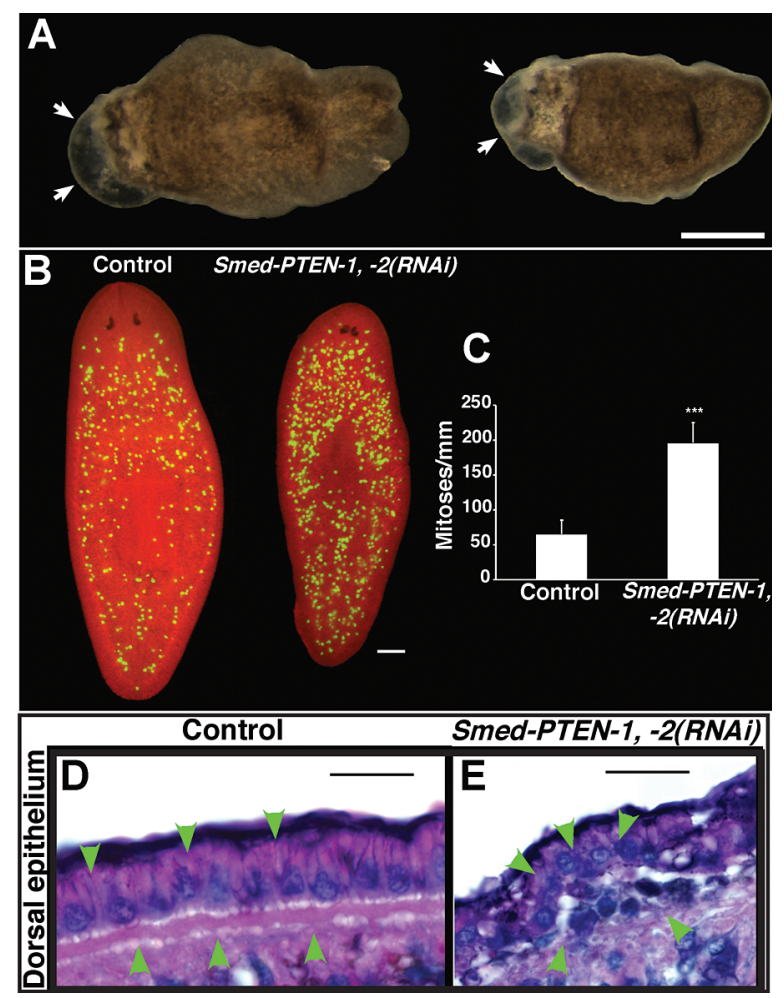

Figure 4. Tumor suppressor function of two PTEN homologs in planarians. (A) Abrogation of gene function via RNAi of the two planarian orthologs of PTEN (Smed-PTEN-1 and -2) results in abnormal outgrowths (white arrows). Bar, $500 \mu \mathrm{m}$. (B) Wholemount immunostaining using $\alpha$-phosphorylated histone $\mathrm{H} 3$ (H3P) antibody in normal and Smed-PTEN-1(RNAi);SmedPTEN-2(RNAi) worms indicates a noticeable increase of cellular proliferation in RNAi-treated animals. $(C) \mathrm{H} 3 \mathrm{P}$-signal quantitation from both control and simultaneous Smed-PTEN-1 (RNAi); Smed-PTEN-2(RNAi) worms. A significant increase $(p<0.0001)$ in mitotic activity was observed in RNAi-treated animals. $(D)$ Paraffin-tissue sections of normal animals stained with hematoxylin and eosin. (Green arrowheads) Columnar epithelium and basement membrane. (E) Hematoxylin and eosin stains of paraffin sections from Smed-PTEN-1(RNAi);Smed-PTEN-2(RNAi). (Green arrowheads) Multiple cell in the epithelium and the disruption of basement membrane. (Data from Oviedo et al. 2008.)

study of TSGs in adult stem cell lineages and have been shown to have tumor suppressor function for PTEN, it will be interesting to test the function of $p 53, R b$, and other "vertebrate-specific" TSGs in these animals. Should the planarian homologs of these genes show tumor suppressor function, we can conclude that their ancestral roles were tumor suppression, which was most likely lost in flies and C. elegans. Finally, because we have shown that these genes evolved before multicellularity, testing gene function of these homologs in a choanoflagellate will also give perspective on their ancestral functions.

Contrary to the testing of $\mathrm{p} 53 / \mathrm{PTEN} / \mathrm{Rb}$ in invertebrates, fly nTSGs need to be further studied for tumor suppressor function in vertebrate ASCs. Only the tissue-specific deletion of the $l g l$ ortholog has been performed and with mixed results (Klezovitch et al. 2004; Vasioukhin 2006). Interesting data have come from a few studies showing that the two mouse Dlgs can rescue the fly $d l g$ mutant (Thomas 
et al. 1997; Humbert et al. 2003). Therefore, from the fly's perspective, the mammalian orthologs are tumor suppressors because they can suppress the tumor formation of a mutant fly. Additionally, planaria, Monosiga, and vertebrates have multiple family members of these proteins, and thus their tumor suppressor function may be hidden until combinations of mutants (or RNAi) are combined. To rigorously test whether the fly nTSGs have tumor suppression in vertebrates, multiple conditional alleles need to be deleted in adult animals and stem cell lineages followednot an easy task in vertebrates. Because we have shown that planarians have a complement similar to that of vertebrates for these three fly nTSGs, testing their function in planarian stem cells is straightforward and would help to resolve whether these genes have ancestral function as tumor suppressors. We also showed that Monosiga has homologs to two of the three fly nTSGs examined in this chapter that can now be tested for tumor suppressor function. Should planarians or Monosiga show a tumor suppressor function for the fly nTSG homologs, this would lend support to the hypothesis that these genes had ancestral tumor suppressor function that was lost in vertebrates. In conclusion, the study of tumor suppression mechanisms in emerging model systems stands to broadly inform the function of TSGs not only in the ontogeny of animal cancers, but also in normal biological functions such as stem cell regulation, regeneration, and tissue homeostasis.

\section{ACKNOWLEDGMENTS}

B.J.P. was funded by Damon Runyon Postdoctoral Fellowship 1888-05. A.S.A. is a Howard Hughes Medical Institute Investigator.

\section{REFERENCES}

Ajioka, I., Martins, R.A., Bayazitov, I.T., Donovan, S., Johnson, D.A., Frase, S., Cicero, S.A., Boyd, K., Zakharenko, S.S., and Dyer, M.A. 2007. Differentiated horizontal interneurons clonally expand to form metastatic retinoblastoma in mice. Cell 131: 378-390.

Aoki, K. and Taketo, M.M. 2007. Adenomatous polyposis coli $(A P C)$ : A multi-functional tumor suppressor gene. J. Cell Sci. 120: $3327-3335$.

Asano, T., Yao, Y., Zhu, J., Li, D., Abbruzzese, J.L., and Reddy, S.A. 2004. The PI 3-kinase/Akt signaling pathway is activated due to aberrant Pten expression and targets transcription factors NF- $\mathrm{KB}$ and c-Myc in pancreatic cancer cells. Oncogene 23: 8571-8580.

Brodsky, M.H., Nordstrom, W., Tsang, G., Kwan, E., Rubin, G.M., and Abrams, J.M. 2000. Drosophila p53 binds a damage response element at the reaper locus. Cell 101: 103-113.

Brusca, R. and Brusca, G. 2003. Invertebrates. Sinauer, Sunderland, Massachusetts.

Carroll, S.L. and Stonecypher, M.S. 2005. Tumor suppressor mutations and growth factor signaling in the pathogenesis of NF1-associated peripheral nerve sheath tumors. II. The role of dysregulated growth factor signaling. J. Neuropathol. Exp. Neurol. 64: 1-9.

Coqueret, O. 2003. New roles for p21 and p27 cell-cycle inhibitors: A function for each cell compartment? Trends Cell Biol. 13: 65-70.

Cuddihy, A.R. and Bristow, R.G. 2004. The p53 protein family and radiation sensitivity: Yes or no? Cancer Metastasis Rev. 23: $237-257$.

de Nooij, J.C., Letendre, M.A., and Hariharan, I.K. 1996. A cyclin-dependent kinase inhibitor, Dacapo, is necessary for timely exit from the cell cycle during Drosophila embryogenesis. Cell 87: 1237-1247.

Derry, W.B., Putzke, A.P., and Rothman, J.H. 2001. Caenorhabditis elegans p53: Role in apoptosis, meiosis, and stress resistance. Science 294: 591-595.

Du, W., Vidal, M., Xie, J.-E., and Dyson, N. 1996. $R B F$, a novel RB-related gene that regulates E2F activity and interacts with cyclin E in Drosophila. Genes Dev. 10: 1206-1218.

Eisenhoffer, G.T., Kang, H., and Sánchez Alvarado, A. 2008. Molecular analysis of stem cells and their descendants during cell turnover and regeneration in the planarian Schmidtea mediterranea. Cell Stem Cell 3: 327-339.

Evers, B. and Jonkers, J. 2006. Mouse models of BRCA1 and BRCA2 deficiency: Past lessons, current understanding and future prospects. Oncogene 25: 5885-5897.

Fei, P., Bernhard, E.J., and El-Deiry, W.S. 2002. Tissue-specific induction of p53 targets in vivo. Cancer Res. 62: 7316-7327.

Gateff, E. 1994. Tumor suppressor and overgrowth suppressor genes of Drosophila melanogaster: Developmental aspects. Int. J. Dev. Biol. 38: 565-590.

Gateff, E. and Mechler, B.M. 1989. Tumor-suppressor genes of Drosophila melanogaster. Crit. Rev. Oncog. 1: 221-245.

Gateff, E. and Schneiderman, H.A. 1974. Developmental capacities of benign and malignant neoplasms of Drosophila. Wilhelm Roux's Arch. Entwicklungsmech. Org. 176: 23-65.

Gatza, C., Moore, L., Dumble, M., and Donehower, L.A. 2007. Tumor suppressor dosage regulates stem cell dynamics during aging. Cell Cycle 6: 52-55.

Hanahan, D. and Weinberg, R.A. 2000. The hallmarks of cancer. Cell 100: $57-70$.

Harris, C.C. 1996. p53 tumor suppressor gene: From the basic research laboratory to the clinic-An abridged historical perspective. Carcinogenesis 17: 1187-1198.

Heemels, M.-T. 2000. Nature insight: Apoptosis. Nature 407: 769.

Huang, H., Potter, C.J., Tao, W., Li, D.M., Brogioli, W., Hafen, E., Sun, H., and Xu, T. 1999. PTEN affects cell size, cell proliferation and apoptosis during Drosophila eye development. Development 126: 5365-5372.

Humbert, P., Russell, S., and Richardson, H. 2003. Dlg, Scribble and $\mathrm{Lgl}$ in cell polarity, cell proliferation and cancer. BioEssays 25: 542-553.

Irwin, M.S. 2004. Family feud in chemosensitvity: p73 and mutant p53. Cell Cycle 3: 319-323.

King, N., Westbrook, M.J., Young, S.L., Kuo, A., Abedin, M., Chapman, J., Fairclough, S., Hellsten, U., Isogai, Y., Letunic, I., et al. 2008. The genome of the choanoflagellate Monosiga brevicollis and the origin of metazoans. Nature 451: 783-788.

Kirkegaard, T., Witton, C.J., McGlynn, L.M., Tovey, S.M., Dunne, B., Lyon, A., and Bartlett, J.M. 2005. AKT activation predicts outcome in breast cancer patients treated with tamoxifen. J. Pathol. 207: 139-146.

Klezovitch, O., Fernandez, T.E., Tapscott, S.J., and Vasioukhin, V. 2004. Loss of cell polarity causes severe brain dysplasia in Lgll knockout mice. Genes Dev. 18: 559-571.

Li, J., Yen, C., Liaw, D., Podsypanina, K., Bose, S., Wang, S.I., Puc, J., Miliaresis, C., Rodgers, L., McCombie, R., et al. 1997. PTEN, a putative protein tyrosine phosphatase gene mutated in human brain, breast, and prostate cancer. Science 275: 1943-1947.

Li, Y. and Prives, C. 2007. Are interactions with p63 and p73 involved in mutant p53 gain of oncogenic function? Oncogene 26: $2220-2225$.

Lowe, S.W. and Sherr, C.J. 2003. Tumor suppression by Ink4aArf: Progress and puzzles. Curr. Opin. Genet. Dev. 13: 77-83.

$\mathrm{Lu}$, W.J. and Abrams, J.M. 2006. Lessons from p53 in non-mammalian models. Cell Death Differ. 13: 909-912.

Marx, J. 2007. Molecular biology. Cancer's perpetual source? Science 317: 1029-1031.

Menut, L., Vaccari, T., Dionne, H., Hill, J., Wu, G., and Bilder, D. 2007. A mosaic genetic screen for Drosophila neoplastic tumor suppressor genes based on defective pupation. Genetics 177: $1667-1677$.

Micchelli, C.A. and Perrimon, N. 2006. Evidence that stem cells reside in the adult Drosophila midgut epithelium. Nature 439: 475-479. 
Moll, U.M. and Slade, N. 2004. p63 and p73: Roles in development and tumor formation. Mol. Cancer Res. 2: 371-386.

Molofsky, A.V., Pardal, R., Iwashita, T., Park, I.K., Clarke, M.F., and Morrison S.J. 2003. Bmi-1 dependence distinguishes neural stem cell self-renewal from progenitor proliferation. Nature 425: 962-967.

Molofsky, A.V., Slutsky, S.G., Joseph, N.M., He, S., Pardal, R., Krishnamurthy, J., Sharpless N.E., and Morrison, S.J. 2006. Increasing $p 16^{I N K 4 a}$ expression decreases forebrain progenitors and neurogenesis during ageing. Nature 443: 448-452.

Moon, N.S., Di Stefano, L., Morris, E.J., Patel, R., White, K., and Dyson, N.J. 2008. E2F and p53 induce apoptosis independently during Drosophila development but intersect in the context of DNA damage. PLoS Genet. 4: e1000153.

Murray-Zmijewski, F., Lane, D.P., and Bourdon, J.C. 2006. p53/p63/p73 isoforms: An orchestra of isoforms to harmonise cell differentiation and response to stress. Cell Death Differ. 13: $962-972$.

Nakahara, Y., Nagai, H., Kinoshita, T., Uchida, T., Hatano, S., Murate, T., and Saito, H. 1998. Mutational analysis of the PTEN/MMACl gene in non-Hodgkin's lymphoma. Leukemia 12: $1277-1280$.

Nedelcu, A.M. and Tan, C. 2007. Early diversification and complex evolutionary history of the p53 tumor suppressor gene family. Dev. Genes Evol. 217: 801-806.

Newmark, P.A. and Sánchez Alvarado, A. 2000. Bromodeoxyuridine specifically labels the regenerative stem cells of planarians. Dev. Biol. 220: 142-153.

Newmark, P.A. and Sánchez Alvarado, A. 2002. Not your father's planarian: A classic model enters the era of functional genomics. Nat. Rev. Genet. 3: 210-219.

Ollmann, M., Young, L.M., Di Como, C.J., Karim, F., Belvin, M., Robertson, S., Whittaker K., Demsky, M., Fisher, W.W., Buchman, A., et al. 2000. Drosophila p53 is a structural and functional homolog of the tumor suppressor p53. Cell 101: 91-101.

Oviedo, N.J., Pearson, B.J., Levin, M., and Sánchez Alvarado, A. 2008. Planarian PTEN homologs regulate stem cells and regeneration through TOR signalling. Dis. Models Mech. 1: 131-143.

Pagliarini, R.A. and Xu, T. 2003. A genetic screen in Drosophila for metastatic behavior. Science 302: 1227-1231.

Reddien, P.W. and Sánchez Alvarado, A. 2004. Fundamentals of planarian regeneration. Annu. Rev. Cell Dev. Biol. 20: 725-757.

Rocco, J.W., Leong, C.O., Kuperwasser, N., DeYoung, M.P., and Ellisen, L.W. 2006. p63 mediates survival in squamous cell carcinoma by suppression of p73-dependent apoptosis. Cancer Cell 9: 45-56.

Rong, Y.S., Titen, S.W., Xie, H.B., Golic, M.M., Bastiani, M., Bandyopadhyay, P., Olivera, B.M., Brodsky, M., Rubin, G.M., and Golic, K.G. 2002. Targeted mutagenesis by homologous recombination in D. melanogaster. Genes Dev. 16: 1568-1581.

Rossi, D.J. and Weissman, I.L. 2006. Pten, tumorigenesis, and stem cell self-renewal. Cell 125: 229-231.

Sánchez Alvarado, A. and Tsonis, P.A. 2006. Bridging the regen- eration gap: Genetic insights from diverse animal models. Nat Rev. Genet. 7: 873-884.

Senoo, M., Pinto, F., Crum, C.P., and McKeon, F. 2007. p63 is essential for the proliferative potential of stem cells in stratified epithelia. Cell 129: 523-536.

Sherr, C.J. and McCormick, F. 2002. The RB and p53 pathways in cancer. Cancer Cell 2: 103-112.

Stevaux, O., Dimova, D., Frolov, M.V., Taylor-Harding, B., Morris, E., and Dyson, N. 2002. Distinct mechanisms of E2F regulation by Drosophila RBF1 and RBF2. EMBO J. 21: 4927-4937.

Sulis, M.L. and Parsons, R. 2003. PTEN: From pathology to biology. Trends Cell Biol. 13: 478-483.

Terzibasi, E., Valenzano, D.R., and Cellerino, A. 2007. The shortlived fish Nothobranchius furzeri as a new model system for aging studies. Exp. Gerontol. 42: 81-89.

The, I., Hannigan, G.E., Cowley, G.S., Reginald, S., Zhong, Y., Gusella, J.F., Hariharan, I.K., and Bernards, A. 1997. Rescue of a Drosophila NF1 mutant phenotype by protein kinase A. Science 276: 791-794.

Thomas, U., Phannavong, B., Müller, B., Garner, C.C., and Gundelfinger, E.D. 1997. Functional expression of rat synapseassociated proteins SAP97 and SAP102 in Drosophila dlg-1 mutants: Effects on tumor suppression and synaptic bouton structure. Mech. Dev. 62: 161-174.

van den Heuvel, S. and Dyson, N.J. 2008. Conserved functions of the pRB and E2F families. Nat. Rev. Mol. Cell Biol. 9: 713-724.

Vasioukhin, V. 2006. Lethal giant puzzle of Lgl. Dev. Neurosci. 28: $13-24$

Wang, W., Warren, M., and Bradley, A. 2007. Induced mitotic recombination of p53 in vivo. Proc. Natl. Acad. Sci. 104: 4501-4505.

Weinberg, R.A. 1995. The retinoblastoma protein and cell cycle control. Cell 81: 323-330.

Wells, B.S., Yoshida, E., and Johnston, L.A. 2006. Compensatory proliferation in Drosophila imaginal discs requires Droncdependent p53 activity. Curr. Biol. 16: 1606-1615.

Wodarz, A. and Nathke, I. 2007. Cell polarity in development and cancer. Nat. Cell Biol. 9: 1016-1024.

Woodhouse, E., Hersperger, E., and Shearn, A. 1998. Growth, metastasis, and invasiveness of Drosophila tumors caused by mutations in specific tumor suppressor genes. Dev. Genes Evol. 207: 542-550.

Xu, T., Wang, W., Zhang, S., Stewart, R.A., and Yu, W. 1995. Identifying tumor suppressors in genetic mosaics: The Drosophila lats gene encodes a putative protein kinase. Development 121: 1053-1063.

Xue, W., Krasnitz, A., Lucito, R., Sordella, R., Van Aelst, L., Cordon-Cardo, C., Singer, S., Kuehnel, F., Wigler, M., Powers, S., Zender, L., and Lowe, S.W. 2008. DLC1 is a chromosome $8 \mathrm{p}$ tumor suppressor whose loss promotes hepatocellular carcinoma. Genes Dev. 22: 1439-1444.

Yilmaz, O.H., Valdez, R., Theisen, B.K., Guo, W., Ferguson, D.O., Wu, H., and Morrison, S.J. 2006. Pten dependence distinguishes haematopoietic stem cells from leukaemia-initiating cells. Nature 441: 475-482. 


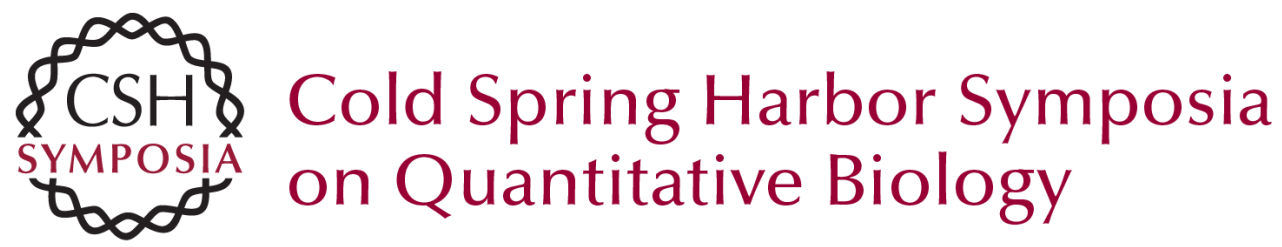

\title{
Regeneration, Stem Cells, and the Evolution of Tumor Suppression
}

\author{
B.J. Pearson and A. Sánchez Alvarado
}

Cold Spring Harb Symp Quant Biol 2008 73: 565-572 originally published online January 15, 2009 Access the most recent version at doi:10.1101/sqb.2008.73.045

References This article cites 67 articles, 17 of which can be accessed free at: http://symposium.cshlp.org/content/73/565.full.html\#ref-list-1

License

Email Alerting Receive free email alerts when new articles cite this article - sign up in the box at the Service top right corner of the article or click here.

To subscribe to Cold Spring Harbor Symposia on Quantitative Biology go to: http://symposium.cshlp.org/subscriptions 